\title{
On the Potential, Feasibility, and Effectiveness of Chat Bots in Public Health Research Going Forward
}

\author{
Stanley Mierzwa ${ }^{1 *}$, Samir Souidi ${ }^{2 *}$, Terry Conroy ${ }^{3}$, Mohammad Abusyed ${ }^{4}$, Hiroki Watarai ${ }^{3}$, \\ Tammy Allen ${ }^{3}$
}

1. Vennue Foundation, Connecticut; John Jay College of Criminal Justice, New York, USA

2. Information Technology, Population Council, New York; Indiana University, Indiana, USA

3. Vennue Foundation, Connecticut, USA

4. Vennue Foundation, Dhaka, Bangladesh

\begin{abstract}
This paper will discuss whether bots, particularly chat bots, can be useful in public health research and health or pharmacy systems operations. Bots have been discussed for many years; particularly when coupled with artificial intelligence, they offer the opportunity of automating mundane or error-ridden processes and tasks by replacing human involvement. This paper will discuss areas where there are greater advances in the use of bots, as well as areas that may benefit from the use of bots, and will offer practical ways to get started with bot technology. Several popular bot applications and bot development tools along with practical security considerations will be discussed, and a toolbox that one can begin to use to implement bots will be presented.

Keywords: Artificial Intelligence; Al; Bots; Chat Bots; Bot Framework; Public Health Technology; Pharmacy Technology

*Correspondence: smierzwa@vennue.org; ssouidi@popcouncil.org

DOI: 10.5210/ojphi.v11i2.9998

Copyright (C2019 the author(s)

This is an Open Access article. Authors own copyright of their articles appearing in the Online Journal of Public Health Informatics. Readers may copy articles without permission of the copyright owner(s), as long as the author and OJPHI are acknowledged in the copy and the copy is used for educational, not-for-profit purposes.
\end{abstract}

\section{Introduction}

An important concept in public health research is that from time to time a new technology or approach to obtain the best possible data, or the application of a new service or tool, will make such an impact that it is then used in education and routinely used in practice for decades. Humans have for many centuries sought ways to automate with machines: in fact, the first humanoid-robot was invented by Arab inventor Al-Jazari, of the Turkish dynasty of Artukids, in 1206 AD. [1] For long-lasting impact, one can look at the use of the body temperature thermometer, which was initially invented for medical use in the late 1800 's; this enormously successful device is still in 
use today. Simply put, it made a positive change on health care that has been sustained over the century since its introduction. Technology is still a catalyst that can be impactful to the level or degree of the body temperature thermometer, and it is up to researchers, practitioners and engineers to continue to experiment and try new ideas and approaches.

This paper will focus on whether the use of a bot (a software application that uses steps or scripts to automate a task and is also known as a web robot or Internet bot), can be beneficial to public health research efforts. Through a variety of toolkits available, chatbots utilize Natural Language Understandings (NLU) services. With NLU, chatbots focus on the use of a conversational interface, one that permits a user to interact using their natural form of speaking. Natural languages (such as English, Hindi, or Portuguese) are used for everyday communication. In contrast to artificial languages, such as programming languages and mathematical notations, natural languages evolve as they pass from generation to generation, and are hard to pin down with explicit rules. [2]

An informal literature review of journal articles found in PubMed Central indicates a lack of practical discussions or thoughts on the topic of bots specifically in use in public health research.

\section{Research Project Background}

A goal for many in the public health sphere is to improve or ensure healthy lives and promote wellbeing for people of all ages. This project explores the use of a simple-to-use chatbot, thus contributing to Goal 3 of the United Nations 2030 Sustainable Development Goals (SDGs). The Good Health and Well-Being SDG is broad, but includes targets aiming at increasing development, training, and retention of health workforces in developing countries. Another goal includes reducing risk associated with the management of health care. Exploring the use of chatbot technology aligns with the SDGs and it is one the authors would explore otherwise, given the increase of its use in the business sector, but it also demonstrates that the United Nations is keeping a keen eye on positive technology trends in industry.

A literature review was performed to determine the popularity of the use of chatbots in public health using key search terms. Tables 1 and 2 outline the results of our search term results focused on chatbots. There are many sources to look for research publications these days, from open-source journals, to Google Scholar and private journals owned by organizations and universities. For the purpose of this paper, we decided to review pertinent articles in the PubMed Central (PMC) housed within the US National Library of Medicine of the National Institutes of Health. The reason for the decision to focus on the PMC was that the authors have published other research papers in journals that would ultimately be linked and housed in the PMC. At present the PMC, which began in the year 2000, now includes over five million full-text records, from thousands of journals. 
Table 1. PMC general search term results.

\begin{tabular}{|l|l|l|}
\hline Search Term (general) & Number of Found Articles & Databases Found \\
\hline "chatbots" & 39 & PubMed; PubMed Central \\
\hline "chat bots" & 40 & $\begin{array}{l}\text { PubMed; PubMed Central; } \\
\text { PubMed Health }\end{array}$ \\
\hline “virtual assistant" & 409 & $\begin{array}{l}\text { Bookshelf; PubMed; PubMed } \\
\text { Central; PubMed Health }\end{array}$ \\
\hline
\end{tabular}

Table 2. PMC search term specific to Title or Abstract results.

\begin{tabular}{|l|l|l|}
\hline $\begin{array}{l}\text { Search Term (Title OR } \\
\text { Abstract) }\end{array}$ & Number of Found Articles & Databases Found \\
\hline "chatbots" & 15 & PubMed; PubMed Central \\
\hline "chat bots" & 2 & PubMed \\
\hline "virtual assistant" & 15 & PubMed \\
\hline
\end{tabular}

As with any new technology solution or technological approach, certain industries will engage with chatbots sooner than others. Much of the initial use or engagement can be due to qualities such as competition, cost savings, or strategic advantage. The case for early adopters of chatbots has been evident in several industries that have much to gain from their use. These include: Hospitality, Banking/Financial, Retail Service Business, and Publishing. [4] A generation of users is eager to use technology in business-related sectors and has moved ahead; however, there is the potential for greater emphasis on the health-related or public health sector. Companies look for self-service ability to make the customer experience more convenient and familiar to consumers whose primary communication platforms have become messaging apps and social networks. More than one in three brands or companies say customers and prospects prefer to complete a transaction or resolve service issues without speaking to a human associate, if possible. When asked which technologies would most improve the customer experience, $40 \%$ of sales and marketing leaders cited Virtual Reality (VR). Thirty-four percent (34\%) believe Artificial Intelligence (AI) will be the biggest game-changer. [7]

According to a study by Juniper Research, the health care and banking industries are primed to see the next largest benefit from the use of chatbots because they handle such large volumes of human interaction. Juniper's research predicts that by 2022, chatbots will save organizations more than $\$ 8$ billion annually worldwide by significantly reducing the amount of time it takes to resolve inquiries. [7]

\section{Methods (Tools)}

As with any software product or software development tools, there are many avenues one can take to solve a problem or implement a solution. In this section we review several of the larger segment bot tools available that have been in use, but it should be noted that the bot development market is 
still developing and different toolsets can emerge. During the time of writing of this paper, several new chatbot developer tools began have begun to gain traction, including Oracle, Rulai and Nuance Communications. The authors utilized this analysis to decide on which chatbot development environment and toolset to create a sample chatbot.

\subsection{Bot Tools Available from IBM Watson}

IBM Watson Assistant (formerly Watson Conversation) offers a comprehensive and developerfriendly portfolio. It marries a technically robust conversational platform to strong developer tools and enables the creation of a wide range of solutions. [8] Forrester Research reports that the IBM Watson Assistant is a leader in their Wave position and that it may be less applicable to device and consumer applications, but more geared toward widespread enterprise device deployments. Links for getting started with building a chatbot with IBM can be found at: https://www.ibm.com/watson/how-to-build-a-chatbot/

\subsection{Bot Tools Available from Google}

Google entered the chatbot market via their AI and Machine Learning products, Dialogflow (formerly API.AI). Forrester reports it as a strong performer via their Wave position. The tool has the reputation of being able to provide a simple user experience in development; it also allows for the rapid creation of a conversational interface. Google has an advantage with their solution because of their vast experience in AI. Forrester says that a pilot-to-production solution can be achieved in a month and a half.

\subsection{Microsoft Bot Framework}

Microsoft has developed and made available a host of tools, starter example packages, and documentation that can be quickly utilized to create chatbots, or integrate bot qualities into existing applications. These toolsets include the ability to create locally available chatbots via Visual Studio, a Bot Builder Software Development Kit (SDK), and an emulator and with C\# support. One can build a bot facility offline, with use of the SDK and test it locally with the available emulator. In addition, Microsoft has launched the Azure Bot Service. The Azure service permits one to create a bot, publish it to Azure or to one's own local web server and, via the public web, allow it to go live and interact with end-users. A user can create his or her own Azure bot and begin traveling through the creation of the hosted box by logging into the Azure portal, selecting AI + Machine Learning from the Azure Marketplace, then selecting Web App Bot.

\subsection{Facebook Bot Toolsets}

Facebook has strong communications in its services and dominates the field because of the many users it has connected. Facebook recently opened up its Messenger service to developers, thus creating the opportunity for programmers to utilize it to create bots. The Facebook Messenger via bot permits multiple people to communicate in a single conversation, adding complexity and advantages. Facebook $\mathrm{M}$ is the service the company plans to push since it includes their Artificial Intelligence and could perhaps provide extremely intelligent and more accurate bots. 


\subsection{Amazon Bot Tool - Amazon Lex}

Amazon Lex is a tool made available by Amazon and is based on the same technology behind their popular Alexa product. The toolset allows developers to create text or voice bots. Integration with such services as Twilio allows users to set up Amazon Lex to respond to SMS messages. Developers can use the Amazon Web Service (AWS) SDKs to build iOS, Android, Java, Python and a host of other programming languages to utilize Lex.

Table 3. Relevant chatbot tools comparison for selection by authors - as of April 2019.

\begin{tabular}{|c|c|c|c|c|c|}
\hline & $\begin{array}{l}\text { IBM } \\
\text { Watson }\end{array}$ & $\begin{array}{l}\text { Dialogic } \\
\text { Flow }\end{array}$ & $\begin{array}{l}\text { Microsoft } \\
\text { Bot } \\
\text { Framework }\end{array}$ & $\begin{array}{l}\text { Facebook } \\
\text { Bot } \\
\text { (Messenger) }\end{array}$ & Amazon Lex \\
\hline $\begin{array}{l}\text { Languages } \\
\text { Supported }\end{array}$ & Over 13 & Over 20 & $\begin{array}{l}\text { Over } 10 \text { (via } \\
\text { LUIS) }\end{array}$ & Many & English only \\
\hline $\begin{array}{l}\text { Year } \\
\text { Developed }\end{array}$ & 2007 & $\begin{array}{l}2010 \\
\text { (Purchased } \\
\text { by Google in } \\
2016 \text { ) }\end{array}$ & 2016 & 2016 & 2010 \\
\hline $\begin{array}{l}\text { Difficulty } \\
\text { Level to } \\
\text { Entry of Use }\end{array}$ & $\begin{array}{l}\text { Medium } \\
\text { (quick to get } \\
\text { started with } \\
\text { tutorials and } \\
\text { Watson } \\
\text { Assistant) }\end{array}$ & $\begin{array}{l}\text { Medium } \\
\text { (quick to get } \\
\text { started with } \\
\text { available } \\
\text { conversation- } \\
\text { al cards) }\end{array}$ & Medium & Low & Low \\
\hline $\begin{array}{l}\text { Require } \\
\text { Internet for } \\
\text { Operation }\end{array}$ & $\begin{array}{l}\text { Yes - must } \\
\text { use IBM } \\
\text { Cloud } \\
\text { Account }\end{array}$ & $\begin{array}{l}\text { Yes - Google } \\
\text { Cloud } \\
\text { Platform } \\
(\mathrm{GCP})\end{array}$ & $\begin{array}{l}\text { Yes - } \\
\text { Microsoft } \\
\text { Azure (can } \\
\text { deploy on } \\
\text { private } \\
\text { servers) }\end{array}$ & Yes & Yes \\
\hline $\begin{array}{l}\text { Platform } \\
\text { Integrations } \\
\text { Available }\end{array}$ & $\begin{array}{l}\text { Watson } \\
\text { Discovery; } \\
\text { Assistant; } \\
\text { Watson } \\
\text { API's }\end{array}$ & $\begin{array}{l}\text { Google } \\
\text { Assistant; } \\
\text { Alexa; } \\
\text { Cortana; } \\
\text { Facebook } \\
\text { Messenger }\end{array}$ & $\begin{array}{l}\text { Azure; } \\
\text { Azure } \\
\text { Cognitive } \\
\text { Services; } \\
\text { Storage; } \\
\text { Facial } \\
\text { Recognition; } \\
\text { Text } \\
\text { Analysis }\end{array}$ & $\begin{array}{l}\text { Chatfuel; } \\
\text { Plugins for } \\
\text { Google and } \\
\text { Bing search; } \\
\text { Video; } \\
\text { Audio }\end{array}$ & $\begin{array}{l}\text { AWS Lambda; } \\
\text { Cognito; Polly }\end{array}$ \\
\hline $\begin{array}{l}\text { SDK } \\
\text { Programming }\end{array}$ & Node.js; & $\begin{array}{l}\text { Node.js; } \\
\text { Python; Java; }\end{array}$ & C\#; Node.js & JavaScript; & $\begin{array}{l}\text { Java; JS; } \\
\text { Python; .NET; }\end{array}$ \\
\hline
\end{tabular}




\begin{tabular}{|c|c|c|c|c|c|}
\hline $\begin{array}{l}\text { Language } \\
\text { Supported }\end{array}$ & & $\begin{array}{l}\text { Go; Ruby; } \\
\text { C\#; PHP }\end{array}$ & & & $\begin{array}{l}\text { Ruby; PHP; } \\
\text { CLI; Go; C++ }\end{array}$ \\
\hline $\begin{array}{l}\text { Example of } \\
\text { External } \\
\text { Entities Bot } \\
\text { Tool Can } \\
\text { Communicate } \\
\text { With }\end{array}$ & $\begin{array}{l}\text { Facebook; } \\
\text { Slack; } \\
\text { Intercom; } \\
\text { Alexa; SMS }\end{array}$ & $\begin{array}{l}\text { Vehicles; } \\
\text { Mobile } \\
\text { Devices; } \\
\text { Speakers, } \\
\text { Phones; } \\
\text { Facebook } \\
\text { Messenger; } \\
\text { Amazon Lex }\end{array}$ & $\begin{array}{l}\text { Skype; } \\
\text { Slack; QnA } \\
\text { Maker; Kik; } \\
\text { Facebook } \\
\text { Messenger; } \\
\text { Office } 365\end{array}$ & $\begin{array}{l}\text { Customer } \\
\text { Chat plugin } \\
\text { for website; } \\
\text { Travel } \\
\text { assistant; } \\
\text { Digital } \\
\text { Marketing; } \\
\text { eCommerce }\end{array}$ & $\begin{array}{l}\text { SalesForce; } \\
\text { MS Dynamics; } \\
\text { Zendesk; } \\
\text { Quickbooks }\end{array}$ \\
\hline $\begin{array}{l}\text { Available } \\
\text { SDKs for iOS } \\
\text { and Android }\end{array}$ & Yes & $\begin{array}{l}\text { Yes; and } \\
\text { many more }\end{array}$ & Yes & $\begin{array}{l}\text { Yes. } \\
\text { Messenger } \\
\text { Platform } \\
\text { APIs }\end{array}$ & Yes \\
\hline $\begin{array}{l}\text { Intended or } \\
\text { Focused Use } \\
\text { Cases }\end{array}$ & $\begin{array}{l}\text { Media; } \\
\text { Advertising; } \\
\text { Finance; } \\
\text { Health; } \\
\text { Education; } \\
\text { IOT; } \\
\text { Customer } \\
\text { Engagement }\end{array}$ & $\begin{array}{l}\text { Customer } \\
\text { Service; } \\
\text { Commerce; } \\
\text { Enterprise } \\
\text { Productivity; } \\
\text { IOT }\end{array}$ & $\begin{array}{l}\text { Virtual } \\
\text { Assistant; } \\
\text { Customer } \\
\text { Care; } \\
\text { Enterprise }\end{array}$ & $\begin{array}{l}\text { eCommerce; } \\
\text { Shopping } \\
\text { List; } \\
\text { Account } \\
\text { Linking }\end{array}$ & $\begin{array}{l}\text { Insurance; } \\
\text { Education; } \\
\text { Field Service; } \\
\text { Tight } \\
\text { integration } \\
\text { with Alexa; } \\
\text { High quality } \\
\text { speech } \\
\text { recognition; }\end{array}$ \\
\hline Cost & $\begin{array}{l}\text { Free to begin } \\
\text { and test. }\end{array}$ & $\begin{array}{l}\text { Free to begin } \\
\text { and test. }\end{array}$ & $\begin{array}{l}\text { Free to } \\
\text { begin and } \\
\text { test. }\end{array}$ & $\begin{array}{l}\text { Free to } \\
\text { begin and } \\
\text { test. }\end{array}$ & $\begin{array}{l}\text { Free to begin } \\
\text { and test. }\end{array}$ \\
\hline
\end{tabular}

\subsection{Prototype Health Research Bot}

The nonprofit Vennue Foundation envisions a prototype chatbot to enhance the quality of care delivered to patients in low-income countries. Vennue's mission is to improve patient health outcomes in the developing world through a unique strategy of pharmacy workforce training. In countries where Vennue has a presence, the local pharmacy is often a patient's only point of access to health care.

The Vennue chatbot will enhance the quality of health care services delivered at the point of dispensing. The envisioned chatbot, planned for development after the writing of this paper, will aid pharmacy personnel by allowing them to use natural language input to ask questions related to pharmacy knowledge they may lack or do not feel confident about. Given the growing burden of diabetes in developing countries, and in view of the frequent visits that diabetes patients make to their pharmacies, Vennue's chatbot will focus on answering diabetes-related queries. 
Worldwide, diabetes is one of the fastest growing chronic diseases, impacting more than 425 million people and directly causing an estimated 1.6 million deaths. [16] Unmanaged diabetes significantly increases the risk for blindness, kidney failure, heart attacks, stroke and lower limb amputation. In low-income countries, the risk for developing these complications is elevated because the prevalence of diabetes is rising more rapidly than in developed countries, and the ratio of physicians to patients is severely inadequate. [14][15]

Current practice standards encourage diabetes self-management education and support (DSMES). To help alleviate the uneven physician-to-patient ratios in the developing world, chatbots have an opportunity to be part of the DSMES by personalizing care and addressing the needs of patients through cost-effective, evidence-based responses and behavioral change interventions.

Diabetes can be treated and its consequences avoided or delayed with diet, physical activity, medication, and regular screening and treatment for complications. A chatbot can empower pharmacy workers to harness these intervention tactics for diabetes patients.

The Vennue chatbot will be designed to enable pharmacy personnel to easily screen patients for diabetes and pre-diabetes, and then make efficient referrals to higher levels of care for follow-up care including clinical diagnosis and treatment. It also may be programmed to answer diabetesrelated questions most frequently asked by pharmacy clients in low-resource settings. In addition, the chatbot may recommend lifestyle modifications and other diabetes consultation points that pharmacy staff can then convey verbally to their patients, immediately at the point of care. Vennue will ensure the chatbot is fit-for-purpose with locally-relevant information and usability.

The creation and results of the Vennue pharmacy chatbot will be discussed in a future research pilot results peer-reviewed paper. The pilot will include data obtained from actual implementation of the chatbot in community-based and hospital pharmacies located in Bangladesh and Nepal, where Vennue's pharmacy workforce training program has demonstrated success.

Serving every day on the frontlines of patient care, pharmacy personnel in the developing world hold remarkable potential to improve health and promote well-being. If equipped with practical training and a locally-relevant chatbot, the pharmacy workforce can fundamentally transform the quality of care and consultation offered to diabetes patients.

After reviewing the different development frameworks available to build a chatbot, the authors decided on the Amazon Lex platform. Although any of the tools discussed in section 3 above would have met the need for this prototype, the authors selected Lex because it was thought to have the lowest barrier to software development and a short development time. In addition, the Lex platform is considered a Strong Performer via the Forrester Wave position and can be seen as third among the solutions analyzed by Forrester.

\subsection{Bot General Workflow Design}

An example of a bot conversation-like flow can be seen in Figure 1. It is important to understand some of the key terms used in bot development: Intent, Utterances, Slots, Prompts, and Fulfillment. Figure 1 below demonstrates the use of these key terms in the context of a sample engagement and 
vision of a chatbot being used. In this sample chatbot, English audio would be used, but with corresponding English text to complement the chatbot conversation or transaction.

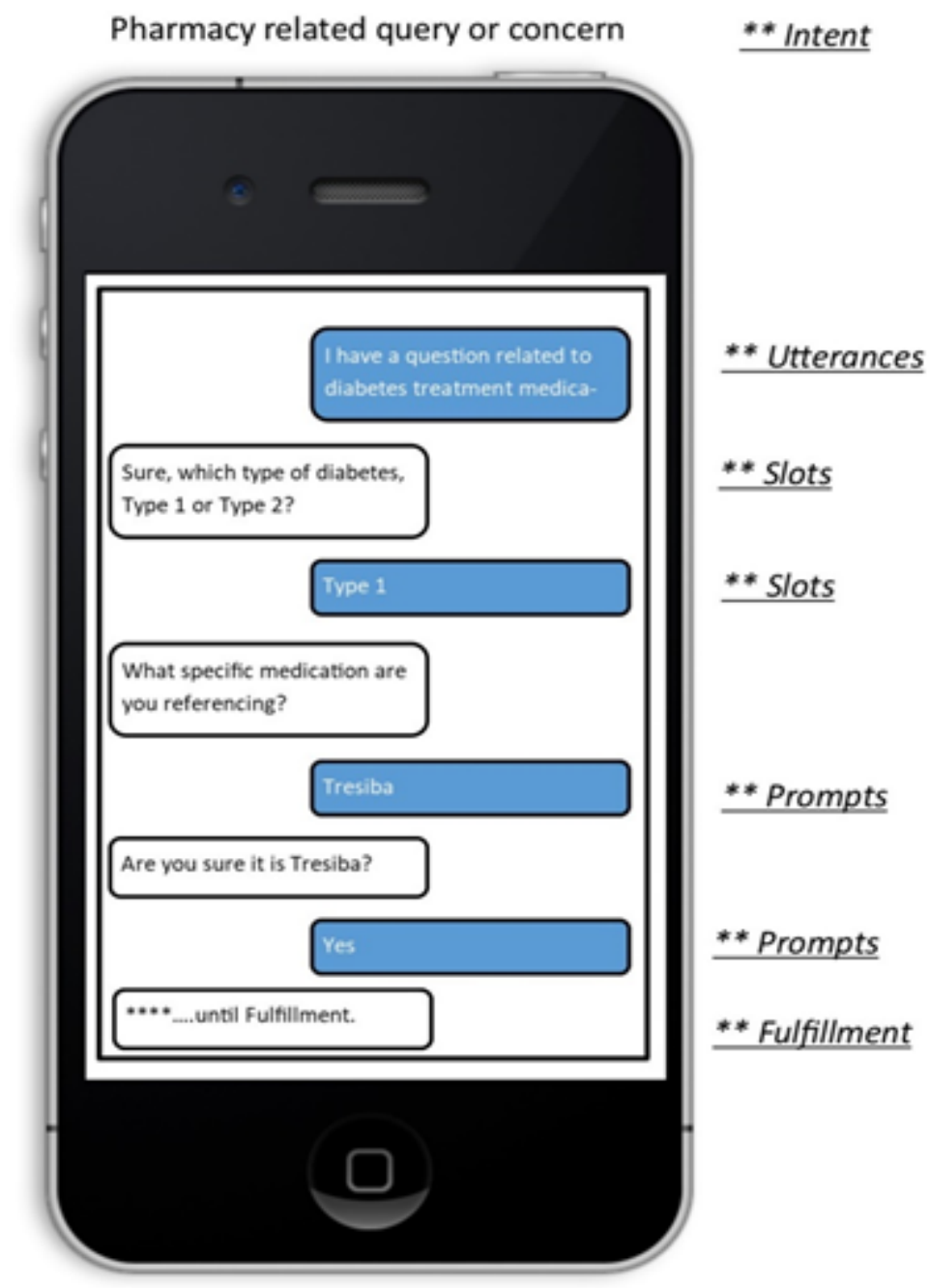

Figure 1. Example of Bot Development Terms.

AWS Lex provides an easy-to-use Amazon console where the user would define bots, intents, and Slot types. The bot performs the automated tasks, such as guiding a customer through ordering a pizza. It is powered by the Automatic Speech Recognition (ASR) and Natural Language Understanding (NLU) capabilities, the same technology powering the Amazon Alexa. The intent represents an action a user would like to perform. A bot can support one or more related intents. In our pizza example, a developer can create a bot that also orders drinks. Intents can utilize zero or more slots or parameters. Adding slots is part of the intent configuration. During runtime of the chatbot application, Amazon Lex prompts the user for specific slot values. The user must provide values for all minimally required slots before Amazon Lex can fulfill the intent. Each slot will include a type, and for this the user creates his or her custom slot types or can use built-in slot types available with Amazon. 


\subsection{Amazon Lex Development Environment}

AWS Lex does not require deep learning or coding experience. AWS Lex could be developed in the AWS console utilizing any web browser or using the AWS Software Development Kit (SDK) combined with the AWS Command Line Interface (CLI) and can be integrated with many programming languages. In our sample development of the pizza ordering chatbot, we used the AWS Console to build an AWS Lex. AWS offers thorough documentation on its website with many how-to examples to develop AWS Lex. The authors followed a step-by-step guide for creating such a chatbot provided by Amazon. [5]

The Amazon console allows the user to define and create these elements in a short time without any coding. The next step is to link the AWS Lex to AWS Lambda where the back-end code handles the AI logic and will interact with the user's input and AWS Lex. AWS Lambda function allows the solution to operate the back-end logic code without provisioning or managing any servers. In our sample application to provide feasibility, the Python language was used in the AWS Lambda function.

The front-end facing application in our feasibility test required coding in order for the mobile device to interact with the AWS Lex. In our example, we used a custom Android JAVA mobile application for the front-end user interface for the "OrderingPizza" chatbot.

\subsection{Development Back-End}

A hosted server with Amazon Web Service (AWS) houses the Amazon Lex developed logic that captures, processes, and provides feedback to the end users who initiated the natural language query. In Figure 2 below the overall component architecture is represented in a simple block diagram. The blocks in Figure 2 cover the front-end Android application presented to the users, the use of an API gateway, and the AWS hosted components. The authors created a simple Lex app to prove feasibility; this mimicked the ability to use the service to order a pizza. [5] The Amazon Lex creation interface service makes it easy to get started with adding Intents and methods to interact with the chatbot. A critical component discovered during this feasibility assessment is that the solution designer needs to give much attention to the data design of the back-end of the chatbot. It is not approached the same way one would approach the development of a relational database - which the authors feel is a much more straightforward mechanism. The chatbot backend data requires much more design thought, on the outcomes of the queries and then to back into the outcomes with the relevant potential queries from an end user. In addition, the technology engineers need to collaborate very closely with the subject matter experts in designing the data to be used - this was clearly apparent during design sessions with the Vennue expert pharmacy education staff. 


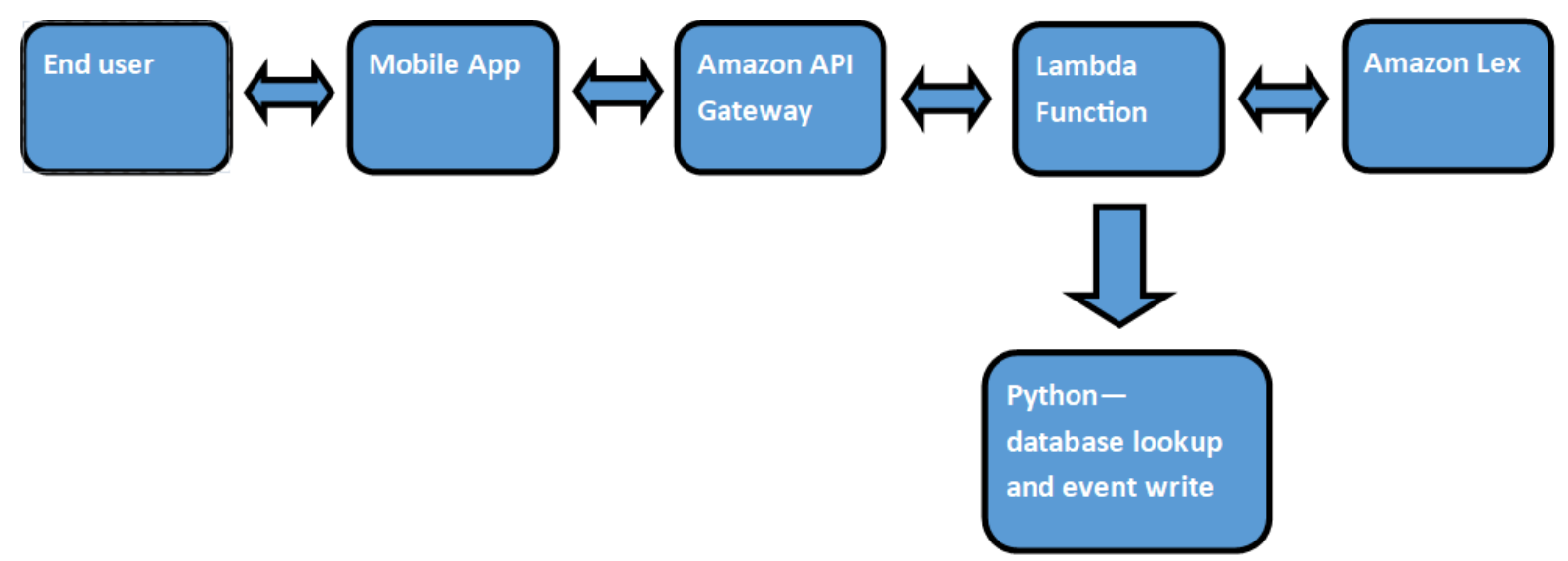

Figure 2. Overall architecture of feasibility chatbot built using the Amazon Lex back-end.

While utilizing the Amazon Lex platform for creation of a chatbot, the user has the ability to create the logic for the application. In our simple example demonstrated in Figure 3, we provide a glimpse into the initiation of engaging with the chatbot via utterances. [5] With the logic builder, the user can continue to add and grow the number of conversational inputs and expand the learning of the application.

\begin{tabular}{|c|c|}
\hline e.g. I would like to book a flight. & $\boldsymbol{\oplus}$ \\
\hline Can I get a $\{$ size $\}\{$ pizzaKind $\}$ pizza & $\boldsymbol{\theta}$ \\
\hline Can I get a $\{$ pizzaKind $\}$ pizza & $\boldsymbol{\theta}$ \\
\hline Can I get a pizza please & $\boldsymbol{\theta}$ \\
\hline I want a $\{$ size $\}$ \{crust\} crust $\{$ pizzaKind\} pizza & $\boldsymbol{\theta}$ \\
\hline I want to order a $\{$ size $\}\{$ pizzaKind\} pizza & $\boldsymbol{\otimes}$ \\
\hline I want to order a \{pizzaKind\} pizza & $\boldsymbol{\otimes}$ \\
\hline I want to order a pizza & $\boldsymbol{0}$ \\
\hline I want to order pizza please & $\boldsymbol{\otimes}$ \\
\hline
\end{tabular}

Figure 3. Sample Amazon Lex logic builder.

In order for a chatbot intent to be fulfilled, slots need to be completed. The number of slots can vary, and there generally will be required a certain amount of fulfilled prior to yielding an effect. In Figure 4 we demonstrate the Amazon Lex Slots screen example, where the sample elements mentioned that need to be fulfilled can be seen. [5] 


\begin{tabular}{|c|c|c|c|c|c|c|c|c|c|}
\hline Priority & & Required & Name & Slot type & & Version & Prompt & Setting & \\
\hline & & & e.g. Location & e.g.AMAZON.US_CITY & $\checkmark$ & & e.g. What city? & & $\oplus$ \\
\hline 1. & $\checkmark$ & $\checkmark$ & crust & Crusts & $\checkmark$ & $1 \nabla$ & What kind of crust would you like? & \# & $\boldsymbol{\theta}$ \\
\hline 2. & $\wedge \vee$ & $\checkmark$ & size & Sizes & $\checkmark$ & $1 \%$ & What size pizza? & * & $\boldsymbol{\theta}$ \\
\hline 3. & ^ & $\checkmark$ & pizzaKind & PizzaKind & $\checkmark$ & $1 \nabla$ & Do you want a veg or cheese pizza? & એ & $\boldsymbol{8}$ \\
\hline
\end{tabular}

Figure 4: Slot definitions.

As part of the process to create the chatbot, there needs to exist a "hook" or connection to the Lex back-end in order to fulfill the request; a sample screen is shown in Figure 5. [5] Lambda functions can be created, or built-in functions may already exist, which will minimize the need to write code.

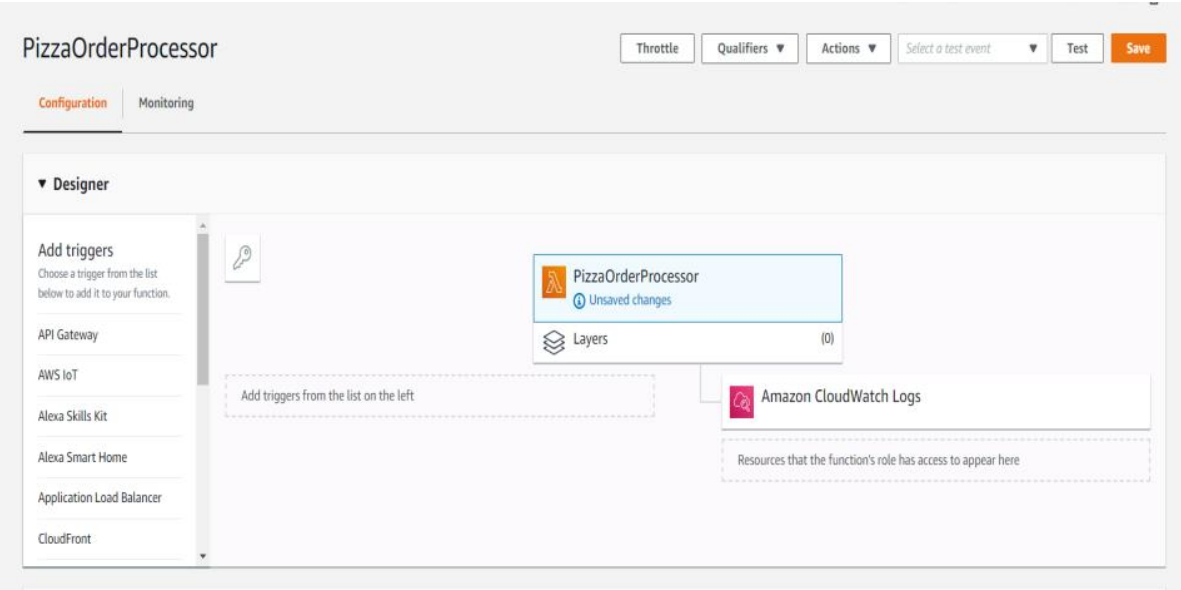

Figure 5: AWS Lambda function.

In a final step, a connection or link is created between the chatbot and the Lambda function. By using the Lambda function, the code or logic lives in the AWS cloud; it is not engaged on the client side of the application. In our example, the connection created to the Lambda function will perform the actual ordering of the pizza, requiring the minimum number of slots fulfilled.

- Fulfillment $\mathbf{\theta}$

- AWS Lambda function $\bigcirc$ Return parameters to client

$\begin{array}{lll}\text { Lambda function } & \text { PizzaOrderProcessor } \\ \text { View in Lambda console } 5 & \checkmark \\ \text { Version or alias } & \text { Latest }\end{array}$

Figure 6: Linking AWS Lex with AWS Lambda function. 


\subsection{Development Front-End}

To complement the Amazon Lex back-end component developed during the feasibility effort, we created a front-end application to provide the interface for the end user. The front-end application was developed for use with Android devices; this was decided because such smartphone or tablet devices are readily available in Bangladesh and Nepal (the locations of the end users who would ultimately utilize the system). Android devices continue to be the dominant mobile device operating system in use in the developing world. [6] The front-end Android application was built using the Java programming language, using the open-source Eclipse Integrated Development Environment (IDE). In testing feasibility, a private web link was provided to end users in Bangladesh and Nepal so that they could install the application, which was not published or made available in the Google Play Store. Interaction or queries sent from the front-end application were submitted to the Amazon Lex back-end chatbot application using the Internet. The access to the back-end was also restricted for use only by this specific feasibility chatbot application.

\section{Cyber and Other Security Threat Concerns}

As with any process or system automation, researchers and information technology engineers must always consider the risks associated with their technology implementation. Given the hypersensitivity and attention given to cyber security in this current day and climate, it would be negligent to not provide such a focus.

In performing an initial cyber security vulnerability assessment, the authors have considered the threats that may come from both remote and local attack surfaces. The remote attack surface, which would include systems that are connected to the Internet and possibly accessible via the web, is a possibility with the introduction of chatbots. The authors used Amazon Lex and Amazon Web Services in the feasibility test application they developed. By their very nature, these web tools and qualities create the potential for cyber threats. The local attack surface would include the Android device that would host the Java-based application that utilizes the Amazon Lex back-end. These Android devices have the potential to be attacked if connected to a mobile or broadband Internet connection and have the ability and potential to download other applications that could introduce vulnerabilities and affect the integrity of the hosted chatbot application. There are many methods for performing a cyber security vulnerability assessment of a system or application. In Tables 4 and 5 below we demonstrate an initial approach one can take to begin a cyber security vulnerability assessment.

\subsection{Cyber Security Threat Mitigations}

The top three cyber threat concerns related to the remote attack surface of the built chatbot feasibility application are:

a. Amazon Lex service hosting chatbot logic availability.

b. Created chatbot system exposed and modified.

c. Access control to AWS chatbot and breach of access. 
Table 4: Remote attack surface (AWS) top threat concerns.

\begin{tabular}{|c|c|c|c|}
\hline & \multicolumn{3}{|c|}{ Likelihood } \\
\hline Consequence & High & Medium & Low \\
\hline High & & & a. Availability \\
\hline Medium & & & b. Exposed \\
\hline Low & & c. Breach & \\
\hline
\end{tabular}

The following are the top three cyber threat concerns related to the local attack surface of the Android application built for chatbot feasibility:

a. Elevation of account privileges (rooting).

b. Inter-process communication - ability to monitor local activity.

c. Lack of availability cause of compromised device.

Table 5: Local attack surface (Android App) top threat concerns.

\begin{tabular}{|c|c|c|c|}
\hline & \multicolumn{3}{|c|}{ Likelihood } \\
\hline Consequence & High & Medium & Low \\
\hline High & & & \\
\hline Medium & c. Compromised & & \\
\hline Low & & a. Elevation & b. Inter-process \\
\hline
\end{tabular}

\section{Discussion}

The term bot has been used frequently in a negative manner. One can often find news in the trade press and on security blogs discussing how bots are affecting elections, stealing information from users unaware that their systems are infected, stealing concert tickets, and so forth. However, it would be beneficial to perhaps change the name of the bots that are doing well. Perhaps we should call them "GBots", "Good Bots", "Botters", or RBots" - something to highlight this new class of positive communication and web robots. The bots discussed in this paper are of the "good" type: those that can be beneficial to companies, researchers, users, and students. In the case of communications, chat bots can be an effective way to increase more intelligent dialog and engagement.

In 2017, 42\% of all Internet traffic was not human, and there were significant year-over-year increases in both bad bot $(+9.5 \%)$ and good bot $(+8.8)$ traffic. [10] The result is that $57.8 \%$ of Internet traffic is human, but more important is the worrisome fact that bad bots take up the greater percentage of traffic.

Initial adoption of chatbots has been rapid among certain business-related industries such as hospitality, banking, and services; the public health field, however, seems to be a bit hesitant in 
moving quickly into tools using artificial intelligence. Further evidence-based research into the uptake of using chatbots or artificial intelligence in public health would benefit the field.

In this feasibility paper, the authors would like to introduce the idea of a consistent cyber security section in future research papers that include a technology component. The benefits of using technology and web-connected technology are well known in research areas. However, it would be prudent to assess the risks associated with using the technology from a cyber threat perspective. Introducing at a minimum a cyber-risk likelihood and consequence analysis would bring confidence to those facilities that utilize the proposed technology solution.

There are areas in the public health sphere that have built chatbots and have had positive experiences. In a study of patients with breast cancer, a chatbot was used as a way to help them by providing support and answers to their concerns during the course of their disease. It was found that using chatbot reminders improved proper use of medications.[18] Researchers also determined that patients appreciated a question of the day provided via the chatbot, and that patients shared information with the chat related to personal and intimate topics, such as sexuality and hair loss. In another research study, which included a self-report survey of physicians on their perceptions of using chatbots in health care, there were positive impressions with the use of introducing chatbots. More than half of physicians surveyed agreed that health care chatbots could help patients better manage their own health (54\%), improve access and timeliness to care (53\%), or reduce travel time to their health care provider (52\%). In addition, $65 \%$ of physicians believed that health care chatbots could improve nutrition or diet, enhance medication or treatment adherence, increase activity or exercise, or reduce stress. [19] This aligns well with the vision of creating the pharmacy chatbot proposed by Vennue Foundation, particularly because of the importance of medication adherence in diabetes care. In the same physician study, challenges were perceived related to the fact that chatbots cannot understand or display human emotion. A majority of the physicians (70\%) expressed their concern of risks associated with health care chatbots for patients. [19]

\section{Conclusion}

Health-related industries must be sure that automation does not create new unintended issues associated with the introduction of new technology. Health care as a whole is in fourth place for the creation of bad bots. [10] $57.58 \%$ of the bots used in health care are good bots, but $24.37 \%$ are bad. This indicates that the health sector is a targeted area for bad bot actors but does not necessarily indicate that the health industry is not doing enough; the health care sector is just a large area that unfortunately produces targeted content.

As with any technology integration or implementation effort, a new bot's purpose must be to make improvements. When introducing bots into public health research, developers will need to ensure they are embarking on the effort to: 1) solve an existing problem, by minimizing the number of steps normally taken; 2) make the end user experience better, faster, or more exact than alternate means in process or that already exist; 3) permit the bot to run on technology easily available to the end user community; and 4) make the interaction with the bot as natural in conversation as possible. 
In focusing on the development of a chatbot for use in public health or pharmacy education, the authors found that building such a solution is feasible given the many options that are available for use by a variety of technology vendors. Although expert programming may not be required for developing the back-end chatbot logic, solution developers will still require technological expertise and comfort with creating solutions in the cloud. In addition, a key aspect in creation of a chatbot will be the data science and design. This was an area that proved more difficult than anticipated when the authors tried to translate the end user requirements into practical use. It is critical that developers work closely with subject matter domain experts when building the database that includes the fulfillment, advice, suggestion, or response for the user of the chatbot.

\section{Limitations}

Many software applications and software development tools are available for bot use; however, we did not do an exhaustive review of every option that could be approached. We rather selected top tier tools that the authors felt were more mature and have a track record of use in various industries.

The next versions of chatbots are evolving and will hopefully include the introduction of multilingual products. At this time, chatbots are mostly focused on or work using the English language; this needs to change.

The authors did not perform a full pilot test with an applied chatbot application as part of this research paper. It is envisioned that a successive actual chatbot pilot will be performed with the Vennue Foundation in Dhaka, Bangladesh and Kathmandu, Nepal. As part of this upcoming pilot, the chatbot would provide a tool for pharmacy staff to utilize the built-in intelligence in the application to obtain diabetes screening information.

\section{Acknowledgments}

We thank Tammy Allen and the team at Vennue Foundation for their excellent collaboration in envisioning whether the use of a chatbot would be appropriate in the pharmacy education environment in the developing world. The Vennue team's insights were invaluable in considering the steps necessary to set up and pilot this first generation public health pharmacy worker prototype chatbot system. We are grateful to Mandip Pokharel of Vennue Nepal and Mirajul Islam Samrat of Vennue Bangladesh for their collaborations at the local pharmacies in-country and their insights on the needs of the pharmacies on the ground. A special hearty thank you to Netania Budofsky for her thorough edit of the research paper, which included questioning certain content to better understand it from a non-expert reader's perspective.

\section{References}

1. Delamater, Natalie. (2018), A Brief History of Artificial Intelligence and How it's Revolutionizing Customer Service Today, SmartMax Software, Inc.

2. Bird, Steven; Klein, Ewan; Loper, Edward. Natural Language Processing with Python, O’Reilly Media, 2009 
3. Buchanan, Bruce G. A. 2005. (Very) Brief History of Artificial Intelligence. AI Mag. 26(4), 53-60.

4. Harrison, Kate L., These 5 Industries Have the Most to Gain from Chatbots, Inc., Oct. 9, 2017

5. Amazon Lex. Developer Guide, Amazon Web Services, 2019

6. Mierzwa, Stan; Souidi, Samir; Austrian, Karen; et al. 2015. Transitioning Customized ACASI Windows.NET Solution To Android Java on Lower-Priced Devices and Technical Lessons Learned. Electron J Inf Syst Dev Ctries. 66(2), 1-11.

7. Chatbot Chatter, More businesses exploring how chatbots can help save money, boost customer service, QP Qualityprogress.com, ASQ, July 2017

8. Koplowitz, Rob; Facemire, Michael, The Forrester New Wave: Conversational Computing Platforms, Q2 2018, The Seven Providers That Matter Most and How They Stack Up, Forrester Research, Inc., April 12, 2018

10. 2018 Bad Bot Report, The Year Bad Bots Went Mainstream, 2018, Distil Networks

11. Cameron, Gillian; Cameron, David; Megaw, Gavin, et al. Best Practices for Designing Chatbots in Mental Healthcare - A Case Study on iHelpr, Proceedings of British HCI 2018, Belfast, UK, 2018

12. Oracle, Can Virtual Experiences Replace Reality. The future role for humans in delivering customer experience, Oracle Corporation, 2016

13. Comendador, Benilda Eleanor; Francisco, Bien Michael; Medenilla, Jeffereson, et. al; Pharmabot: A Pediatric Generic Medicine Consultant Chatbot, Journal of Automation and Control Engineering, Vol 1. No. 2 April 2015

14. Diabetes Fact Sheet. https://www.who.int/news-room/fact-sheets/detail/diabetes, World Health Organization, October 2018

15. IDF Diabetes Atlas. https://www.diabetesatlas.org, International Diabetes Federation, $8^{\text {th }}$ Edition, 2017

16. Gilles R. Dagenais, Hertzel C. Gerstein, Xiaohe Zhang. 2016. Variations in Diabetes Prevalence in Low-, Middle-, and High-Income Countries: Results From the Prospective Urban and Rural Epidemiological Study, American Diabetes Association. Diabetes Care. 39(5), 780-87. PubMed https://doi.org/10.2337/dc15-2338

17. Achieving the health-related MDGs. It takes a workforce! https://www.who.int/hrh/workforce_mdgs/en/, World Health Organization, April 2010 
18. Chaix, Benjamin; Bibault, Jean-Emmanuel; Pienkowski, Arthur; et al. 2019. When Chatbots Meet Patients: One-Year Prospective Study of Conversations Between Patients with Breast Cancer and a Chatbot. JMIR Cancer. 5(1), e12856. PubMed https://doi.org/10.2196/12856

19. Palanica, Adam; Flaschner, Peter; Thommandram, Anirudh; et al. 2019. Physicians' Perceptions of Chatbots in Health Care: Cross-Sectional Web-Based Survey. J Med Internet Res. 21(4), e12887. PubMed https://doi.org/10.2196/12887 